\title{
High incidence of vertebral and non-vertebral fractures in the OSTRA cohort study: a 5-year follow-up study in postmenopausal women with rheumatoid arthritis
}

\author{
M. Vis • E. A. Haavardsholm • P. Bøyesen • \\ G. Haugeberg • T. Uhlig • M. Hoff • A. Woolf • \\ B. Dijkmans • W. Lems • T. K. Kvien
}

Received: 4 December 2009 /Accepted: 29 September 2010/Published online: 13 January 2011

(C) The Author(s) 2011. This article is published with open access at Springerlink.com

\begin{abstract}
Summary A 5-year follow-up study was performed in female RA patients with established disease looking at vertebral fractures, scored on spinal X-rays, and nonvertebral fractures. We found a high incidence rate of vertebral and non-vertebral fractures in these patients compared to population-based studies.

Introduction The aim of this study is to investigate the incidence of vertebral and non-vertebral fractures over a 5year period in a cohort of postmenopausal patients with established rheumatoid arthritis (RA).
\end{abstract}

M. Vis $(\bowtie) \cdot$ B. Dijkmans $\cdot$ W. Lems

Department of Rheumatology and Jan van Breemen Institute, VU

University medical center,

Postbus 7057, 1007MB, Amsterdam, The Netherlands

e-mail: marijn.vis@gmail.com

E. A. Haavardsholm • P. Bøyesen • T. Uhlig • T. K. Kvien Department of Rheumatology, Diakonhjemmet Hospital, Oslo, Norway

G. Haugeberg

Sørlandet Hospital,

Kristiansand, Norway

M. Hoff

Department of Rheumatology, St. Olavs Hospital, NTNU, Trondheim, Norway

A. Woolf

Royal Cornwall Hospital,

Truro, UK

T. K. Kvien

Faculty of Medicine, University of Oslo,

Oslo, Norway
Methods One hundred and fifty female patients with established RA were included into the OSTRA cohort. The cohort was assessed at baseline and at 5 years for incident vertebral and non-vertebral fractures. Spinal X-rays were taken at baseline and at follow-up and scored using the semi-quantitative method according to Genant.

Results At 5 years, 102 patients (68\%) were examined and included in the present analysis. At baseline, the mean age was 61 years, disease duration 17 years, body mass index $25.5 \mathrm{~kg} / \mathrm{m}^{2}$ and $65 \%$ of the patients were rheumatoid factor positive. Fifteen percent were treated with bisphosphonates, $25 \%$ received calcium supplementation and $20 \%$ vitamin-D supplementation at baseline. During the 5-year follow-up, a total of 16 patients out of 102 patients $(16 \%)$ had a new non-vertebral fracture [annual incidence of $3.2(95 \% \mathrm{CI}$ 1.8-5.5) per 100 patients/year]. In 18 patients out of 97 patients $(19 \%)$, new vertebral fractures were identified on spinal X-ray [annual incidence of 3.7 (95\% C.I. 2.2-5.8) per 100 patients/year].

Conclusions We found a high incidence of vertebral and non-vertebral fractures in a cohort of women with established RA compared to population-based studies.

Keywords Female $\cdot$ Fractures $\cdot$ Osteoporosis $\cdot$ Rheumatoid arthritis Vertebral fractures

\section{Introduction}

Osteoporosis is a well-known extra-articular feature of rheumatoid arthritis (RA). Bone mineral density (BMD) is decreased in patients with RA $[1,2]$. The clinical endpoint of osteoporosis, fractures are also more prevalent in RA 
patients compared to the general population [3-5]. Reasons for this decreased BMD and increased prevalence of fractures in RA include among others inflammation, reduced physical activity and corticosteroid use [2]. Almost all data regarding osteoporosis in RA are generated from cross-sectional studies. Longitudinal studies are scarce, especially studies with a focus on fractures. Recently, Van Staa et al. reported that in a large case-control study, the risk of fractures was about 1.5 times higher in RA patients than in healthy controls [4]. In this study, only clinical fractures were assessed and spinal X-rays were not performed routinely to identify asymptomatic vertebral fractures. However, these asymptomatic fractures are also associated with an increased risk of new fractures and with an increased morbidity [6, 7].

The OSTRA group (OSlo, TRuro, Amsterdam) is an international collaboration investigating osteoporosis in RA. Five years ago, the OSTRA group performed a study in postmenopausal patients with RA and found that radiological joint damage (total Larsen score) was associated with a low BMD and vertebral fractures [8].

To further clarify the association between RA and osteoporosis, we performed a 5-year follow-up assessment of this cohort. The main objective of this 5-year follow-up study was to evaluate the incidence of vertebral and nonvertebral fractures in female postmenopausal RA patients.

\section{Patients and methods}

\section{Patients}

All 150 patients from the original study were eligible to participate in the follow-up study. The inclusion and exclusion criteria for the baseline study have previously been described in detail [8]. In short, in each of three centres, general rheumatology clinics in Oslo (Norway), Truro (UK) and Amsterdam (The Netherlands), 50 female patients were consecutively enrolled. The patients included were 5070 years old and fulfilled the American College of Rheumatology (formerly American Rheumatism Association) 1987 revised classification criteria for RA. The disease duration of all patients was $\geq 5$ years [9]. In total, 102 patients of the original cohort consented to a follow-up assessment (33 from Oslo, 34 from Truro and 35 from Amsterdam). The main reasons for not participating in the follow-up study were as follows: 15 moved away from the hospital area, five suffered from severe co-morbidity, eight had died and 20 did not participate for unknown reasons or could not be contacted. The baseline characteristics of the 102 patients who had a follow-up measurement did not differ from the characteristics of all the 150 patients at baseline and of those patients $(n=42)$ that dropped out (lowest $p=0.282$; data not shown).
Demographics and medical history

Data at follow-up were collected from interviews, clinical examination, questionnaires and patient's medical records and included height, weight, calcium intake, history of falls (number of falls during the last year and cause) and fractures (anatomical site and cause), current and previous use of anti-osteoporotic [anti-resorptive therapy (ART) and hormone replacement therapy (HRT)] and disease-modifying anti-rheumatic drugs (DMARDs), and history of corticosteroid use (previous and current use, cumulative amount over the past 5 years, use of $7.5 \mathrm{mg}$ for $>6$ months and number of months on corticosteroids). Physical disability was assessed by means of the Health Assessment Questionnaire (HAQ; 20 items, score range $0-3$, with higher scores indicating worse disability) [10].

\section{Disease activity}

Measures of RA disease activity were assessed with visual analogue scales $(0-100 \mathrm{~mm})$ of pain and patient's global disease activity; 28 tender and swollen joint counts, and acute phase reactants [the erythrocyte sedimentation rate $(\mathrm{ESR} ; \mathrm{mm} / \mathrm{h})$ and $\mathrm{C}$-reactive protein (CRP; mg/L), both measured with standardised local measurement techniques]. The modified 28 joints disease activity score (DAS-28) was calculated according to published guidelines [11]. Joint scores were performed by experienced rheumatology nurses in Oslo and Truro and in Amsterdam by a physician (MV). The mean ESR and CRP were calculated based on all available measurements during the 5-year follow-up.

\section{Fractures}

Non-vertebral Non-vertebral fractures and their cause were assessed at 5-year follow-up by interview and were validated by checking available data (radiology reports and chart review). Low-energy traumatic fractures (i.e. a fall from standing height) were regarded as osteoporotic fractures.

Vertebral All spinal X-rays were taken according to local protocol; the same protocol was used at baseline and followup. Lateral radiographs of the spine were scored according to the semi-quantitative method described by Genant et al. [12]. Scoring was performed individually by two trained observers (MV and WL) and consensus both at baseline and followup was obtained in cases of discrepancies between both observers. Follow-up radiographs were scored blinded for the baseline image, and the results were subsequently compared to the baseline $\mathrm{X}$-rays and scores to see if new 
Table 1 Characteristics of the 102 patients with RA included in the 5-year follow-up na not applicable

${ }^{a}$ Used for at least 1 month during the 5-year follow-up period

${ }^{\mathrm{b}}$ Using at follow-up

${ }^{\mathrm{c}} \mathrm{T}$-scores at either total hip and/or vertebral spine

\begin{tabular}{|c|c|c|c|}
\hline & & Baseline & Follow-up \\
\hline Age, years & Mean (SD) & $61(6)$ & na \\
\hline Disease duration, years & Median (range) & $17(6-25)$ & na \\
\hline IgM-RF positive $(>25 \mathrm{U} / \mathrm{ml})$ & $n(\%)$ & $67(65)$ & $67(65)$ \\
\hline Joint erosions present, patients & $n(\%)$ & $85(83)$ & $85(83)$ \\
\hline BMI, $\mathrm{kg} / \mathrm{m}^{2}$ & Mean (SD) & $25.5(5)$ & $26.0(5)$ \\
\hline HAQ & Mean (SD) & $1.48(0.62)$ & $1.59(0.89)$ \\
\hline \multicolumn{4}{|l|}{ Corticosteroids } \\
\hline Ever use & $n(\%)$ & $65(64)$ & na \\
\hline Use (during follow-up) & $n(\%)$ & na & $58(57)^{\mathrm{a}}$ \\
\hline Months used (during follow-up) & Mean (SD) & na & $43.8(25.4)$ \\
\hline$\geq 7.5 \mathrm{mg}$ for $\geq 6$ months (during follow-up) & $n(\%)$ & na & $18(32)$ \\
\hline \multicolumn{4}{|l|}{ Anti-osteoporosis medication } \\
\hline Anti-resorptive treatment & $n(\%)$ & $15(15)$ & $31(31)^{\mathrm{b}}$ \\
\hline Hormone replacement treatment & $n(\%)$ & $31(30)$ & $0(0)^{\mathrm{b}}$ \\
\hline Calcium supplementation & $n(\%)$ & $41(40)$ & $51(50)^{\mathrm{b}}$ \\
\hline Vitamin-D supplementation & $n(\%)$ & $28(27)$ & $43(42)^{\mathrm{b}}$ \\
\hline Vitamin-D and calcium supplementation & $n(\%)$ & $25(24)$ & $40(39)$ \\
\hline \multicolumn{4}{|l|}{ DMARD treatment (during follow-up) } \\
\hline Methotrexate & $n(\%)$ & na & $66(65)^{\mathrm{a}}$ \\
\hline Duration, months & Mean (SD) & na & $49.2(21.7)$ \\
\hline Sulfasalazine & $n(\%)$ & na & $28(27)^{\mathrm{a}}$ \\
\hline Duration, months & Mean (SD) & na & $40.3(25.2)$ \\
\hline TNF inhibitors & $n(\%)$ & na & $20(20)^{\mathrm{a}}$ \\
\hline Duration, months & Mean (SD) & na & $18.2(11.3)$ \\
\hline Other & $n(\%)$ & na & $44(43)^{\mathrm{a}}$ \\
\hline \multicolumn{4}{|l|}{ Disease activity } \\
\hline DAS-28 & Mean (SD) & $5.4(1.3)$ & $3.6(1.2)$ \\
\hline $\mathrm{ESR}, \mathrm{mm} / \mathrm{h}$ & Median (range) & $27(2-85)$ & $18(2-93)$ \\
\hline CRP, mg/L & Median (range) & $11(0-175)$ & $5(9-72)$ \\
\hline Mean ESR, mm/h & Mean (SD) & na & $20.9(11.8)$ \\
\hline Mean CRP, mg/L & Mean (SD) & na & $12.6(10.9)$ \\
\hline \multicolumn{4}{|l|}{ Osteoporosis/osteopenia $^{c}$} \\
\hline Osteoporosis (T-score $<-2.5$ ) & $n(\%)$ & $36(35)$ & na \\
\hline Osteopenia (T-score $<-1.5$ and $>-2.5$ ) & $N(\%)$ & $26(26)$ & na \\
\hline \multicolumn{4}{|l|}{ Fractures } \\
\hline Vertebral (Genant) & $n(\%)$ & $15(25)$ & $32(33)$ \\
\hline Non-vertebral & $n(\%)$ & $24(24)$ & $35(35)$ \\
\hline
\end{tabular}

vertebral fractures were detected. A fracture was scored as an incident vertebral fracture if it was not present at baseline or if there was a significant increase in loss of height (more than 20\%) in a vertebra which was already fractures at baseline.

\section{Ethics}

The study protocol was approved by the local medical ethical committees of the three centres and all patients gave written informed consent.

\section{Statistical analysis}

Patients with incident fractures (vertebral or non-vertebral fractures) were compared to those not having a new fracture with regard to demographic variables, clinical variables and BMD using two-sided $t$ tests for continuous variables and chisquare tests for counts. The incidence of patients with fractures was expressed per 100 patients/year with $95 \%$ confidence intervals (CI). Possible predictors of incident vertebral and non-vertebral fractures were subsequently examined in a multivariate logistic regression analysis. The 


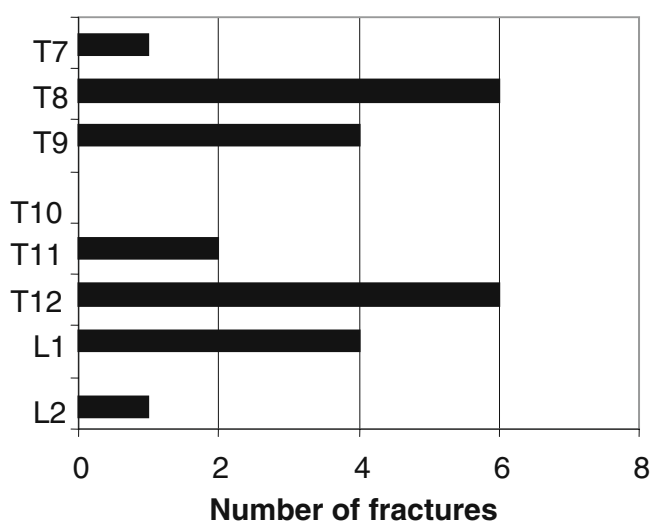

Fig. 1 Distribution of new vertebral fractures

criteria for entering independent variables in the logistic regression analysis were a $p$ value $<0.2$ in the univariate analysis and a supposed clinical relevance for the dependent variable. We were able to build a prediction model with only significant covariates by using backward stepwise elimination of the least significant covariate. All statistical analyses were performed using SPSS (Chicago, IL, USA) version 15.0.

\section{Results}

\section{Patient characteristics}

The clinical characteristics of the 102 patients included in this study are presented in Table 1. At baseline, the patients had a mean (SD) age of 61 (6) years with a median (range) disease duration of $17(6-25)$ years, $83 \%$ of the patients had erosive disease and $65 \%$ patients were rheumatoid factor positive.
The characteristics of the patients during follow-up are shown in Table 1. During follow-up, 58 (57\%) patients used corticosteroids for a mean (SD) duration of 43.8 (25.4)months. ART was used by $15 \%$ of the patients at baseline, and during follow-up an additional 16 patients $(16 \%)$ started with ART. Calcium and vitamin-D supplementation were ever used by $50 \%$ and $42 \%$, respectively, for some time during the follow-up period. HRT was used by $31(30 \%)$ patients at baseline, but was discontinued by all patients by the end of the study.

Incident non-vertebral fractures

A total of 18 patients reported 22 fractures. Two patients had fractures due to high-energy trauma (traffic and skiing accident). Thus, 16 (16\%) patients had 17 osteoporotic fractures. Fractures were reported at the following anatomical sites: upper $\operatorname{arm}(n=3)$, wrist $(n=4)$, hip $(n=3)$, upper leg $(n=$ $2)$, ankle $(n=2)$, ribs $(n=2)$ and pubic bone $(n=1)$. The annual incidence of patients with non-vertebral fractures in our study was 3.2 (95\% CI 1.8-5.5) per 100 patients/year.

\section{Incident vertebral fractures}

A total of 97 patients had lateral spine X-rays available for evaluation. In a total of 18 (19\%) patients, 22 new vertebral fractures were identified. All incident fractures occurred in vertebrae which were normal at baseline. Three patients suffered more than one fracture. Most fractures as expected were identified in the mid-thoracic and thoraco-lumbar regions (Fig. 1). Fifteen of the 18 patients $(83 \%)$ had at least a new grade 2 vertebral fracture. The annual incidence

Table 2 Demographics and disease variables for patients with and without new vertebral and non-vertebral fractures baseline or follow-up

\begin{tabular}{|c|c|c|c|c|c|c|c|}
\hline & & \multicolumn{3}{|c|}{ Vertebral fracture } & \multicolumn{3}{|c|}{ Non-vertebral fracture } \\
\hline & & Yes (18) & No (79) & $p$ & Yes (16) & No (86) & $p$ \\
\hline Age, years & Mean (SD) & $61(6.5)$ & $60(5.8)$ & 0.49 & $62(5.0)$ & $60(6.1)$ & 0.20 \\
\hline Disease duration, years & Mean (SD) & $17(8.7)$ & $17(10.5)$ & 0.95 & $18(8.7)$ & $17(10.7)$ & 0.70 \\
\hline IgM-RF positive & $N(\%)$ & $9(50)$ & $24(30)$ & 0.278 & $10(62)$ & $57(67)$ & 0.77 \\
\hline BMI, $\mathrm{kg} / \mathrm{m}^{2}$ & Mean (SD) & $25.1(3.8)$ & $25.1(4.0)$ & 0.96 & $24.7(2.9)$ & $25.6(5.1)$ & 0.27 \\
\hline HAQ & Mean (SD) & $1.56(0.35)$ & $1.4(0.72)$ & 0.30 & $1.4(0.75)$ & $1.5(0.68)$ & 0.79 \\
\hline Use of corticosteroids & $N(\%)$ & $14(78)$ & $43(54)$ & 0.04 & $11(69)$ & $47(54)$ & 0.30 \\
\hline Use of ART during follow-up & $N(\%)$ & $7(39)$ & $24(30)$ & 0.49 & $10(62)$ & $21(24)$ & 0.002 \\
\hline BMD spine, $\mathrm{g} / \mathrm{cm}^{2}$ at baseline & Mean (SD) & $0.981(0.193)$ & $1.159(0.516)$ & 0.12 & $0.969(0.132)$ & $1.151(0.585)$ & 0.08 \\
\hline BMD hip, $\mathrm{g} / \mathrm{cm}^{2}$ at baseline & Mean (SD) & $0.843(0.138)$ & $0.840(0.165)$ & 0.96 & $0.751(0.108)$ & $0.858(0.159)$ & 0.003 \\
\hline DAS-28 at baseline & Mean (SD) & $5.2(0.7)$ & $4.7(1.2)$ & 0.06 & $4.8(1.2)$ & $4.8(1.2)$ & 0.89 \\
\hline Mean ESR, mm/h & Mean (SD) & $22.3(13.3)$ & $20.1(11.5)$ & 0.49 & $21.7(13.6)$ & $20.8(11.6)$ & 0.80 \\
\hline Mean CRP, mg/L & Mean (SD) & $15.7(8.0)$ & $11.3(8.1)$ & 0.07 & $12.5(6.4)$ & $12.7(11.7)$ & 0.82 \\
\hline Vertebral fracture at baseline & $N(\%)$ & $1(5)$ & $11(15)$ & 0.067 & $5(31)$ & $19(22)$ & 0.44 \\
\hline Non-vertebral fracture at baseline & $N(\%)$ & $8(44)$ & 15 (19) & 0.02 & $4(25)$ & $10(11)$ & 0.12 \\
\hline
\end{tabular}


rate for a new morphometric vertebral fracture was 3.7 (95\% CI 2.2-5.8) per 100 patients/year.

In total, $32(32 \%)$ patients had either a new vertebral or a new non-vertebral fracture.

Differences in patients with and without vertebral and non-vertebral fractures

On average, patients with a new non-vertebral fracture had a lower BMD at baseline compared to patients without a new non-vertebral fracture. This was significant only for the baseline BMD at the hip $(p<0.05)$. In the group of patients with a new non-vertebral fracture, more patients used ART than in the group without a new non-vertebral fracture $(62 \%$ versus $24 \%, p<0.05)$.

When comparing patients with and without incident vertebral fractures, there were significantly more patients using corticosteroids (78\% versus 54\%) in the patients with a new vertebral fracture during follow-up $(p<0.05)$. Patients with new vertebral fractures had also suffered significantly more non-vertebral fractures at baseline $(p<0.05)$, but seemed to have less vertebral fractures at baseline $(p=0.067)$. There was also a trend for a higher disease activity (mean CRP during follow-up and DAS-28 at baseline) in the patients with a new vertebral fracture compared to patients without a new vertebral fracture (Table 2).

Of the patients who were osteopenic at baseline, seven (19\%) sustained a new vertebral fracture and six (17\%) a new non-vertebral fracture during follow-up. In the group of osteoporosis patients, there were seven $(27 \%)$ new vertebral and seven $(27 \%)$ new non-vertebral fractures during follow-up.

Possible risk factors for incident fractures

In the multivariate logistic analysis, we identified BMD at the total hip as an independent predictor for incident nonvertebral fractures. BMD of the spine, ever steroid use, vertebral fractures and non-vertebral fractures at baseline were entered into the model, but were eliminated as not significant (Table 3). Non-vertebral fractures at baseline were an independent predictor of new vertebral fractures. BMD of the spine, mean CRP over the follow-up period, DAS-28 at baseline and ever steroid use were entered into the model but were eliminated (Table 3). All regression models were corrected for centre.

\section{Discussion}

In this 5-year follow-up study of postmenopausal women with established RA, we found a high incidence of vertebral and non-vertebral fractures. Baseline non-vertebral fractures were an independent predictor of new vertebral fractures and new non-vertebral fractures were independently predicted by baseline BMD at the hip. This is the first study to study incident non-vertebral fractures and morphometric vertebral fractures in RA in a single study. These data are also unique because of the duration of follow-up (5 years).

In total, $19 \%$ of the patients had a new vertebral fracture during the 5-year follow-up, corresponding to an annual incidence of 3.7/100 patients/year. Because this is an observational study, we have no data from a control group to compare this annual incidence. Comparison with other historical cohorts is possible. In the European Prospective Osteoporosis Study (EPOS), a study of fractures in the general population of 50 years and older, the annual incidence rate of morphometric vertebral fractures in females was 1.07 per 100 patient years [13]. Mean age (63 years) for these patients is comparable to our study. In another study by Nevitt et al., the annual incidence of morphometric fractures was $0.8 / 100$ patient years. This study assessed fractures in subjects 65 years and older from the general population [14]. Although comparisons between studies should be considered with caution, these studies give a clear indication of the high incidence rate of vertebral fractures in our study. The vertebral fractures we found were also predominantly moderate and severe fractures (grades II and III). There are two studies which performed a longitudinal study on radiological detected vertebral fractures. Ørstavik et al. found 6.7 incident deformities per 100 patient years in a group of 255 female RA patients (mean age 54.3 years) during a mean follow-up of 2.3 years [15]. This study, however, did not use vertebral spine X-rays but morphometric X-ray absorptiometry; this different technique may
Table 3 Multivariate analyses of incident fractures

\begin{tabular}{lcll}
\hline & $B$ & OR $(95 \% \mathrm{CI})$ & $p$ value \\
\hline Non-vertebral fractures & & & \\
BMD total hip $\left(1.0 \mathrm{~g} / \mathrm{cm}^{2}\right)$ & -5.6 & $0.003(0.001-0.42)$ & 0.019 \\
Constant & 2.8 & 16.1 & 0.133 \\
Vertebral fractures & & & 0.029 \\
Non-vertebral fracture at baseline & 1.21 & $3.4(1.3-9.6)$ & 0.54 \\
Constant & 0.6 & 1.8 & \\
\hline
\end{tabular}


explain the higher incidence rate of vertebral fractures in this otherwise comparable study. In the other study, Katsumitsu et al. [16] found new vertebral fractures in $19(16 \%)$ patients out 112 patients followed for 4 years. This percentage is comparable to the percentage of vertebral fractures found in our study during 5 years (19\%). Unfortunately, no data are given on the mean disease duration and mean age of the included RA patients.

In our study, we also found a high frequency of nonvertebral fractures. When comparing our annual incidence of 3.1 per 100 patients/year with the incidence from the female population in the EPOS study (1.9/100 patient years), it is considerably higher. The EPOS is a study investigating limb fractures in men and women aged 50 to 79 years [17]. Finigan et al. also found an incidence 1.9 of new vertebral fractures per 100 patient years in a 10-year follow-up populationbased study. Three hundred and sixty-seven female patients were included into this study with an age (64.6 years) at baseline which is comparable to our cohort [18]. Few studies have investigated the incidence of clinical fractures in RA patients. In a large database study by van Staa et al., they identified an increased risk of fractures of 1.5 for all fractures in RA patients compared to healthy controls [4]. This study included all clinical fractures, also including clinical vertebral fractures. Nampei et al. found in a cohort of 209 RA patients (86\% female, mean age 60 years) an incidence of patients with new fractures of $11.5 / 100$ patient years [19]. This is a very high incidence, but this study investigated all patients with pain suspicious of a fracture very thoroughly (including MRI) for fractures, which could very well explain the high incidence of fractures in this study.

In our study, we found few risk factors for new fractures. Our study only revealed well-known risk factors for new vertebral fractures and new non-vertebral fractures, respectively baseline non-vertebral fractures and BMD of the hip at baseline. We did not find any specific RA-related factors to be predictors for new fractures. Mean CRP and baseline DAS- 28 showed a trend to be increased in patients with a new vertebral fracture (Table 3), but were not independent predictors of future vertebral fractures.

Our study has several limitations. We performed measurements at baseline and at follow-up at 5 years. This is a quite long period and measurements like DAS-28 at baseline and follow-up will probably not properly reflect the fluctuation of the disease activity during that period. This could explain why we found no associations between fractures and disease activity. Another reason for not finding an association could be that joint scores were performed by different investigators, which can cause some variability in measurements. However, we also did not find an association with objective disease activity measures like CRP and ESR. Finally, our studied population might also be too small to find risk factors in rheumatoid arthritis for a multifactorial disease like osteoporotic fractures. Another limitation of having only measurements at baseline and 5 years is that we were not able to properly investigate the relationship between timing of the use of anti-resorptive treatment and new fractures especially vertebral fractures. It could be very likely that bisphosphonates were started after a fracture occurred and this is probably the reason is why we did not find a protective effect of bisphosphonates for example.

In conclusion, in our study we found a high incidence rate of vertebral and non-vertebral fracture rates during a follow-up of 5 years in patients with established RA compared to the general population.

\section{Conflicts of interest None.}

Open Access This article is distributed under the terms of the Creative Commons Attribution Noncommercial License which permits any noncommercial use, distribution, and reproduction in any medium, provided the original author(s) and source are credited.

\section{References}

1. Haugeberg G, Uhlig T, Falch JA et al (2000) Bone mineral density and frequency of osteoporosis in female patients with rheumatoid arthritis: results from 394 patients in the Oslo County Rheumatoid Arthritis register. Arthritis Rheum 43:522-530

2. Lems WF, Dijkmans BA (1998) Should we look for osteoporosis in patients with rheumatoid arthritis? Ann Rheum Dis 57:325-327

3. Cooper C, Coupland C, Mitchell M (2000) Rheumatoid arthritis, corticosteroid therapy and hip fracture. Ann Rheum Dis 54:49-52

4. van Staa TP, Geusens P, Bijlsma JW et al (2006) Clinical assessment of the long-term risk of fracture in patients with rheumatoid arthritis. Arthritis Rheum 54:3104-3112

5. Ørstavik RE, Haugeberg G, Mowinckel P et al (2004) Vertebral deformities in rheumatoid arthritis: a comparison with populationbased controls. Arch Intern Med 23:420-425

6. Burger H, Van Daele PLA, Algra D et al (1994) Vertebral deformities as predictors of non-vertebral fractures. BMJ 309:991-992

7. Lindsay R, Silverman SL, Cooper C et al (2001) Risk of new vertebral fracture in the year following a fracture. JAMA 285:320-323

8. Lodder MC, Haugeberg G, Lems WF et al (2003) Radiographic damage associated with low bone mineral density and vertebral deformities in rheumatoid arthritis: the Oslo-Truro-Amsterdam (OSTRA) collaborative study. Oslo-Truro-Amsterdam (OSTRA) Collaborative Study. Arthritis Rheum 49:209-215

9. Arnett FC, Edworthy SM, Bloch DA et al (1998) The American Rheumatism Association 1987 revised criteria for the classification of rheumatoid arthritis. Arthritis Rheum 31:315-324

10. Fries JF, Spitz P, Kraines RG et al (1980) Measurement of patient outcome in arthritis. Arthritis Rheum 23:137-145

11. van Gestel AM, Prevoo ML, t Hof MA et al (1996) Development and validation of the European League Against Rheumatism response criteria for rheumatoid arthritis. Comparison with the preliminary American College of Rheumatology and the World Health Organization/International League Against Rheumatism Criteria. Arthritis Rheum 39:34-40 
12. Genant HK, Wu CY, van Kuijk C et al (1993) Vertebral fracture assessment using a semiquantitative technique. J Bone Miner Res 8:1137-1148

13. EPOS study group (2002) Incidence of vertebral fracture in Europe: results from the European Prospective Osteoporosis Study (EPOS). J Bone Miner Res 17:716-724

14. Nevitt MC, Cummings SR, Stone et al (2005) Risk factors for a first-incident radiographic vertebral fracture in women at least 65 years of age: the study of osteoporotic fractures. J Bone Miner Res 20:131-140

15. Orstavik RE, Haugeberg G, Uhlig T et al (2005) Incidence of vertebral deformities in 255 female rheumatoid arthritis patients measured by morphometric X-ray absorptiometry. Osteoporos Int 16:35-42
16. Katsumitsu A, Tadamasa H, Hiroya S et al (2006) Risk factors for vertebral fracture in menopausal or postmenopausal Japanese women with rheumatoid arthritis: a cross-sectional and longitudinal study. J Bone Miner Res 24:118-124

17. Ismail AA, Pye SR, Cockerill WC (2002) Incidence of limb fracture across Europe: results from the European Prospective Osteoporosis Study (EPOS). Osteoporos Int 13:565-567

18. Finigan J, Greenfield DM, Blumsohn A et al (2008) Risk factors for vertebral and nonvertebral fracture over 10 years: a population-based study in women. J Bone Miner Res 23:75-85

19. Nampei A, Hashimoto J, Koyanagi J et al (2008) Characteristics of fracture and related factors in patients with rheumatoid arthritis. Mod Rheumatol 18:170-176 